\title{
Rigid and flexible ureteroscopy (URS/RIRS) management of paediatric urolithiasis in a not endemic country
}

\author{
Stefania Ferretti ${ }^{1}$, Monica Cuschera ${ }^{1}$, Davide Campobasso ${ }^{2}$, Claudia Gatti $^{3}$, Riccardo Milandri ${ }^{1}$, \\ Tommaso Bocchialini ${ }^{1}$, Elisa Simonetti ${ }^{1}$, Pietro Granellil, Antonio Frattini ${ }^{2}$, \\ Umberto Vittorio Maestroni ${ }^{1}$ \\ ${ }^{1}$ Urology Unit, University-Hospital of Parma, Italy; \\ ${ }^{2}$ Urology Unit, Hospital of Guastalla, Azienda USL-IRCCS of Reggio Emilia, Italy; \\ ${ }^{3}$ Paediatric Surgery Unit, University-Hospital of Parma, Italy.
}

\begin{abstract}
Summary Introduction: In the last years due to miniaturization of endoscopic instruments and percutaneous surgery, endourology has become very popular in paediatric urinary stone managment. We reported our single-centre experience in retrograde endoscopic procedures in children. Results and complications of URS/RIRS are discussed.

Materials and methods: We retrospectively reviewed our experience in patients $\leq 16$ years old affected by urinary stones who underwent URS/RIRS procedures performed by two surgeons with expertise in endourology. A total of 30 renal Units (RUs) underwent endoscopic procedures (URS, RIRS or both). Surgical complications according to the ClavienDindo's classification and stone-free rate were evaluated at 3 months follow-up. Success of URS was defined as stone-free status after single procedure while RIRS success rate was considered as presence of residual stone fragments smaller than $4 \mathrm{~mm}$ at first procedure.

Results: The mean age of our patients was 8 years, range 216 years. A total of 30 renal units (RUs) underwent 40 endourological procedures (23 URS and 17 RIRS; 10 children underwent both procedures at the same time). 17/30 (56.6\%) RUs were pre-stented before surgery. The stone-free status was achieved in 23/30 renal units treated, with a $76.6 \%$ success rate. The remaining 7 patients had residual stones greater than $4 \mathrm{~mm}$ and underwent further treatments. After a second surgery the stone-free rate turned out to be $93.3 \%$ (28/30 renal units).

Conclusions: Rigid and flexible ureteroscopy (URS/RIRS) is a reliable technique for treatment of $<2 \mathrm{~cm}$ urinary stones in paediatric age group. It shows low rate of major complications and promising results in terms of stone-free rate.
\end{abstract}

KEY WORDS: Flexible ureterescopy; Paediatric stone disease; RIRS; Laser lithotripsy.

Submitted 8 September 2021; Accepted 27 October 2021

\section{INTRODUCTION}

The management of paediatric urolithiasis is nowadays more common in the current urologic practice. It is well known as an endemic problem in developing countries, but the incidence of nephrolithiasis in the paediatric population has been steadily growing also in the European countries and in North America (1). During the last years, we have seen a higher incidence of paediatric stones disease: $5-13 \%$ in Western countries and 20\% in Saudi Arabia, Egypt, Sudan, India and Thailand (2). All ages of the childhood, and both genders can be affected equally. Reasons are not clear and multiple factors have been suggested like obesity, changes in dietary habits with increased sodium intake, decreased calcium and water assumption and increasing use of fructose and antibiotics (1-3). The treatment is based on similar techniques as for adults, especially extracorporeal shock wave lithotripsy (SWL) and endourological techniques (semirigid ureteroscopy - URS, flexible retrograde intrarenal surgery - RIRS and percutaneous nehrolithotomy - PNL).

The aim of stone management in children should be complete stone clearance, prevention of new stone formation and re-growth, preservation of renal function, control of urinary tract infection, minimal invasiveness, less anesthesia, less radiation exposure, as few as possible surgical drainage procedures and correction of both the anatomic abnormalities and the underlying metabolic disorders (4). Decision making for treatment strategies depends on the number, size, location, composition of stones and anatomy of the urinary system. In the last ten years due to miniaturization of endoscopic instruments and increased experience into retrograde and percutaneous treatment on adult patients, endourology has become the best approach to treat urinary stones in children. RIRS is effective and has become a good option in the treatment of renal stones $<2$ $\mathrm{cm}$ (4-6). The aim of this study is to report our single-centre experience in paediatric stone management with retrograde endoscopic procedures. Results and complications of URS/RIRS are discussed.

\section{MATERIALS AND METHODS}

We retrospectively reviewed our experience in patients $\leq 16$ years old affected by urinary stones who underwent URS/RIRS procedures performed by two surgeons (AF and SF) with expertise in endourology. Twenty-eight patients were studied (19 male and 9 female), 2 of these suffering from bilateral renal stones and treated in separate surgical sessions. A total of 30 renal Units (RUs) underwent endoscopic procedures (URS, RIRS or both). 
The data were collected in collaboration with the Paediatric Surgery Unit at the University Hospital of Parma from January 2009 to May 2017. Local ethical committee approval was obtained for data collection (protocol number: 490/2019/OSS*/AOUPR). All patients underwent preoperative blood tests, urine culture and in $78.5 \%$ of patients (22/28) a low dose "flash" CT-scan without contrast medium was performed before surgery; all patients underwent abdominal ultrasound. Further evaluation (eg. MRI-scan, voiding cistouretrography, renal scintigraphy) were performed only in selected cases. Stone localization and size measurement were performed by non-contrast helical Computed Tomography (CT) scanning or Kidneyureter-bladder (KUB) radiographs/ultrasound exams.

We considered 2 types of stone parameters: a. maximum length of the stones and $b$. surface area using Tiselius' formula ( $\mathrm{SA}=$ length $\times$ width $\times \pi \times 0.25$ ).

Procedures were performed with ultrathin $6.5 / 7 \mathrm{Fr}$ or $8 \mathrm{Fr}$ semirigid ureteroscope (Storz, Germany) or flexible ureteroscope X-Flex2 7.5Fr (Storz, Germany) in RIRS.

A 9.5Fr ureteral access sheath $(20 \mathrm{~cm}$ or $28 \mathrm{~cm}$ length Cook, USA) was inserted when possible. In no case active dilation of distal ureter was performed during surgery due the potential risk of secondary ureteral stricture or vesicoureteral reflux. Stone fragmentation was achieved by 35 W holmium:YAG laser lithotripsy device (Quanta System, Italy). A laser fiber of 200 or 273 micron was used. X-ray was administered by Digital X-Ray EV Endurance Philips with internal protocol reducing doses to $1 / 4$ of total adult's dose. Protective diaphragms were used for thyroid gland and genitalia because of the potential radiation risk. A routine preoperative antibiotic prophylaxis with a third generation cephalosporin or amoxicillin/clavulanic acid was administered to all patients and all procedures were performed under general anesthesia. After surgery, a ureteral catheter, mono-J or double-J was left in place based on the duration of the procedure, degree of ureteral edema and/or residual fragments. Generally, the JJstent's strings were left if we planned to leave the stent for up 10 days. A bladder catheter was left for 24-48 hours in all patients. The removed stones underwent spectrometric analysis. Surgical complications according to the ClavienDindo's classification and stone-free rate (assessed by abdominal ultrasound and "flash" helical CT-scan in doubtful cases) were evaluated at 3 months follow-up. All postoperative outcome data of patients referred from other centers (53\%) were verified by telephone interview with the local physician and/or the parents. Success of URS was defined as stone-free status after single procedure while RIRS success rate was considered as presence of residual stone fragments smaller than $4 \mathrm{~mm}$ (Clinical Insignificant Residual Fragments) at first procedure.

We reported the global success rate of both procedures at first step and second step (re-do surgery).

\section{Results}

From January 2009 to May 2017, 28 children with urolithiasis were managed at our centre. Ten preschoolage children (1-5 yrs) and 18 school-age children (6-16 yrs). The mean age of our patients was 8 years, range 216 years. Urological comorbidities included: 7 recurrent urinary infections, 6 ureteropelvic junction dysplasias, 2 megaureters, 1 distal ureteral substenosis, 1 renal double district, 2 vesicoureteral refluxes, 5 observed metabolic disorders (3 idiopathic hypercalciurias, 1 cystinuria, 1 hyperoxaluria). Relevant general comorbidities: 2 infant cerebral palsies, 1 thalassemia, 1 autism, 1 Lowe syndrome, 1 amelogenesis imperfecta. Previous urological surgery: 3 orchidopexies, 2 circumcisions, 2 pyeloplasties, 1 varicocele, 1 bulking agent injection for vesicoureteral reflux, 1 pyelolithotomy, 4 extracorporeal shock wave lithotripsies, 1 percutaneous nephrostomy; other procedures: 1 bowel resection for acute ischemic disease, 1 laparoscopic cholecystectomy, 1 removal of thoracic angiofibroma and 1 stabilization of the hips and femurs.

A total of 30 renal units (RUs) underwent 40 endourological procedures (23 URS and 17 RIRS; 10 children underwent both procedures at the same time). In some cases a second look surgery was necessary. At the end of all surgical sessions 25 URS and 24 RIRS were recorded. The average stone area was $1.15 \mathrm{~cm}^{2}$ and the range of maximum stone diameter was 5-24 mm. Preoperative Grade I and II hydropnephrosis was present in 15 RUs (11 grade I, 4 grade II). No Grade III or IV was reported. 17/30 (56.6\%) RUs were pre-stented before surgery (16 double JJ and 1 mono J); 15/17 in emergency for pain/fever (first access to paediatric surgery unit), 2/17 for ineffective previous ureteroscopy in other hospital; 13/30 RUs were not pre-stented at first procedure with positive surgical results; the age ranged from 5 to 16 years and the procedures were 8 simple URS and 5 URS/RIRS. In 12/24 RIRS we inserted 9.5 Fr ureteral access sheat- UAS (10/17 at first RIRS and 2/7 at second RIRS). None of the patients experienced access failure at surgery. Demographic and Preoperative data are shown in Table 1. Considering the total amount of procedures (surgery and redo surgery) 37 indwelling ureteral stents (29 double JJ and 8 mono J) were placed.

\section{Table 1.}

Demographic and preoperative data.

\begin{tabular}{|l|c|}
\hline Data & Patients \\
\hline Age (years) & $8(2-16)$ \\
\hline Sex (male/female) & $19 / 9$ \\
\hline Lateralization (n) & 15 \\
Right & 11 \\
Left & 2 \\
Bilateral & \\
\hline Stone location (\%) & 35 \\
Ureter & 28 \\
Pelvis & 16 \\
Cl & 9 \\
CM & 7 \\
CS & 5 \\
UPJ & $5-24$ \\
\hline Stone size: max lenght (mm) & 1,15 \\
\hline Stone surface area (cm²) & $15(50)$ \\
\hline Pre-operative hydronephrosis (RUs), $n(\%)$ & 17 \\
\hline Pre-operative stent insertion for RUs, $n(\%)$ & $1(5.8)$ \\
MJ & $16(94.2)$ \\
DJ & \\
\hline
\end{tabular}


Table 2.

Redo surgery data.

\begin{tabular}{|l|c|}
\hline Data & Patients \\
\hline Age (years) & $7(2-12)$ \\
\hline Sex (male/female) & $5 / 2$ \\
\hline Pre-operative stenting (n) & $7 / 7$ \\
\hline Surgical procedures (n) & $5 / 7$ \\
RIRS & $2 / 7$ \\
URS\&RIRS & $5.5(3-14)$ \\
\hline Mean hospital-stay (days) & $3 / 7$ \\
\hline Post-operative stent insertion (n) & $4 / 7$ \\
MJ & $9(2-35)$ \\
DJ & $5 / 7(71.4 \%)$ \\
\hline Mean stent-indewelling time (days) \% & \\
\hline Stone-free status (n pts) \% & \\
\hline
\end{tabular}

Table 3.

Operative and postoperative outcomes.

\begin{tabular}{|l|c|}
\hline Data & Patients \\
\hline Type of procedures (total $n$ ) & 25 URS \\
\hline UAS insertion (n) & 24 RIRS \\
\hline Operative Time (minutes) & $12 / 24$ \\
\hline X-ray exposure (seconds) & $77.7(20-140)$ \\
\hline Postoperative stent insertion for RUs, $n$ (\%) & $14(2-72)$ \\
DJ & 37 \\
MJ & $29(78.3)$ \\
\hline Ureteral stent removal (days) & $8(21.7)$ \\
\hline Hospital stay (days) & $14(2-57)$ \\
\hline Stone composition (RUs), $n$ (\%) & $5(2-13)$ \\
Ca oxalate & 30 \\
Mixed & $17(56.6)$ \\
Ammonium urate & $4(13.4)$ \\
Calcium phosphate & $3(10)$ \\
Unknown & $3(10)$ \\
Cystine & $2(6.7)$ \\
\hline Stone free rate after $1^{\circ}$ procedure (RUs), $n$ (\%) & $1(3.3)$ \\
\hline Stone free rate after 2 ${ }^{\circ}$ procedure (RUs), $n$ (\%) & $23(76.6)$ \\
\hline Complications (RUs), n (\%) & $28(93.3)$ \\
Clavien I & $4(10.8)$ \\
Clavien II & 2 \\
Clavien III & 2 \\
Clavien IV & 0 \\
Clavien V & 0 \\
& 0 \\
\hline
\end{tabular}

The average time of indwelling stenting after first procedure was 14 days with a range of 2 to 57 days. During the procedure, the mean X-Ray exposure time was 14 seconds, with a range of 2-72 seconds. The duration of operations was calculated from intubation to awakening from the anesthesia owing to different OR logs in that period of time. Average time recorded was 78 minutes with a range of 30 to 140 minutes. The mean hospital stay was 5 days with a range of 2-13 days. stones were composed of calcium oxalate $(56.6 \%)$, calcium phosphate $(10 \%)$, ammonium urate $(10 \%)$, cystine $(3.3 \%)$ and mixed (13.4\%).

The stone-free status was achieved in 23/30 renal units treated, with a $76.6 \%$ success rate. The remaining 7 patients had residual stones greater than $4 \mathrm{~mm}$ and underwent further treatments. After a second surgery the stone-free rate turned out to be $93.3 \%$ (28/30 renal units). In patients needing a surgical reoperation, the second procedure was performed after an average time of 74 days (14 days-330 days). The two failed children underwent renal SWL after 5 months while the last one required a MicroPERC ${ }^{\circledR}$ (PolyDiagnost) for a minor calyx's residual stone one year later (Table 2). According to the Clavien-Dindo's classification, complications occurred in $10,8 \%$ of all 37 renal units treated. In particular, minor complications (grade I and II) consisted of: fever during postoperative time (1 case), vomiting ( 1 patient with PEG), hematuria (1 case) and urinary tract infection (1 case). No major complications (grade III and IV) occurred. No patient needed blood transfusion. Two patients died because of their concomitant medical conditions (cerebral palsy and Lowe syndrome) 2 and 5 years after surgery, respectively. One patient affected by hypotrophic kidney before surgery one year later underwent nephrectomy. Operative and post-operative outcomes are summarized in Table 3.

\section{Discussion}

Nowadays minimally invasive endoscopic techniques such as URS/RIRS and mini-PNL - are considered the best approach for the treatment of paediatric urolithiasis in terms of efficacy and safety due to the miniaturization of the surgical instruments, the increasing incidence also in industrialized countries and the experience with adult patients (4-6). The patients we studied showed a high proportion of comorbidities: $42.8 \%$ urological malformations, $25 \%$ urinary tract infections, $25 \%$ non-urologic diseases and, finally, $17.8 \%$ metabolic disorders. These data demonstrate that paediatric nephrolithiasis is related to urologic malformations or infections and metabolic disorders (1-3). As many authors have pointed out, thanks to the introduction of flexible ureteroscopy it has become possible to treat both lower and upper urinary tract stones (7-9). This technique is suitable even when stones are located in tricky sites, such as lower calyces, or in case of renal and skeletal malformations where SWL are not recommended. Extracorporeal shock wave lithotripsy (SWL) was introduced twenty years ago, the 2019 European Guidelines on paediatric urolithiasis suggested for pelvic stones less than $2 \mathrm{~cm}$ this approach and RIRS or microPNL as secondary treatment options.

Moreover, the stone-free rate is significantly affected by various factors. When the stone size increases (> 1.5/2 $\mathrm{cm}$ ), the need for additional sessions increases in parallel. SWL was found to be less effective for caliceal stones and particularly for lower caliceal stones. Several studies reported stone-free rates varying between 50\% and 62\% (10). Ather et al. also assessed that stone-free rate decreases with increasing stone size and in case of lower calyxes stones (11). Although SWL is a non-invasive technique, some stones would require multiple sessions with the need of general anaesthesia in younger children. For the treatment of our patients we used semi-rigid ureteroscopy in 34\% of cases, flexible instruments in $32 \%$ of cases and both in 34\% of cases (using semi-rigid to start the procedure and flexible to complete it). Compared to adult URS, paediatric URS is still performed in a much smaller group of patients with an over- 
all risk of complications or failure slightly higher than in adults $(6,12)$. The importance of expertise in endourological procedures (rigid/flexible) was essential to improve the success rate. For example, Kucukdzman et al. reported an incidence of $3.7 \%-12 \%$ in paediatric ureteroscopy series for proximal ureteral stone migration (13). Today, in many hospital realities, this aspect is not considered as a complication due to the possibility to manage the push-up of the stone in the renal cavities. To share knowledge between paediatric and adult surgeon (twin-surgeon model) as a tutor at the beginning of the learning curve could be the answer for higher and quickly levels of performances (14). The difficulty in performing ureteroscopy in children younger than 3 or 5 years (due to the smaller ureteral diameter) is well known (1516). Our data show that 4 out 13 not pre-stented patients were up to five years old and no one experienced access failure to ureters. Moreover, it is important to use ultrathin semirigid ureteroscopes for negotiation the access to the ureteral meatus with light hydro-dilation and, at the same time, permitting a passive ureteral dilation before retrograde procedures with or without ureteral access sheath $(17,2)$. The use of UAS is under debate in the paediatric population for the potential risk of ureteral damage. In our experience, 12/24 RIRS were performed with UAS without complications; we believe that in renal stone smaller than $1 \mathrm{~cm}$ is not mandatory the use of ureteral access sheath like in adults. Berettini et al. reported a good experience in 13 pts weighing $<20$ $\mathrm{kg}$ who underwent to RIRS with UAS; all patients presented 2 weeks before surgery. In $93.8 \%$ of cases, the UAS was inserted without complications and, at a mean follow up of 22 months, no long-term post-surgical complication was reported (15). Erkurt et al. reported the positioning of UAS in only $61.5 \%$ of cases and an incidence of 2 ureteral wall injuries due to sheath but without related complications at long-term follow up; in this population UAS before RIRS was inserted in $94.1 \%$ of pre-stented children and only $50 \%$ of non-stented patients (9). Chu et al. found a decreased operative time, re-do surgery rates, improved SFR and reduced risk of ureteral injury with the use of UAS in children pre-stented before RIRS (16). At the end of the procedures, all patients had a stent with a general good tolerance except for 2 patients (7\%) who complained severe dysuria. Four children held the stent for more than one month while awaiting the second surgery; the prolonged time was due to complex clinical situations (cerebral palsy, two prolonged antibiotic therapies for other diseases). Stent placement after endoscopic procedures is a controversial issue. The device allowed reduction of pain owing to local edema and also limited the risk of infection due ureteral obstruction for residual fragments. At the same time, however, it required a readmission to operating room for removal. In our experience, children older than 14 years were subjected to outpatient stent removal procedure with a mild premedication; in all the other cases a short deep sedation was required. We are convinced of the importance of leaving self-removal strings for less ten days of indwelling time. Considering our patients, the stones showed an average surface area of $1.15 \mathrm{~cm}^{2}$ in accordance with the data from the literature (6-9).
The stones that required a second procedure were located in the pelvis and lower renal calices; the initial stone burden and multiple locations played an important role in re-do surgery. Lower pole stones seem to be more difficult to manage in particular when the stone is in an anterior calyx. Several Authors pointed out the necessity of multiple SWL sessions for obtaining a stone-free status in kidney stones in more than $70 \%$ of children. We believe that ureteroscopy reduced the risk of additional general anesthesia sessions, allowed the possibility to treat different stone localizations and to reduce X-ray exposure respect to SWL multiple procedures $(18,19)$. The data analyzed in our department show $76.6 \%$ stonefree rate after the first surgery and $93.3 \%$ stone-free rate after second look procedure in accordance with the literature which presents a variable stone-free rate between $77 \%$ and $100 \%(6-9,17,2)$. Complications were evaluated according Clavien-Dindo's classification with 10.8\% of patients showing grade I and II complications. No ureteral perforation was observed differently from a perforation rate of 2 to $7.3 \%$ reported in the literature in paediatric URS series (13). Endoscopic management of urinary stones is increasingly used also in paediatric population. The limitations of our study include its retrospective nature and the small series of patients that is due to the location of our hospital (North Italy) in a nonendemic geographic area for stones. On the other hand, our series is a homogeneous cohort of patients treated by only two surgeons with long experience in adult endourology (AF and $\mathrm{SF}$ ) rather than by surgeons with different levels of expertise influencing stone-free success and complication rate. A larger population-based trial would be essential for confirming these preliminary data.

\section{Conclusions}

Nephrolithiasis in paediatric patients is a relevant disease both in terms of incidence, which is sharply increasing; relapses are quite frequent and can reduce patient's quality of life. Evaluation of associated conditions such as urologic malformations, urinary tract infections, metabolic disorders, turns out to be fundamental in order to perform an early diagnosis and finally schedule a tailored treatment, with the least possible impact for the child. The choice of treatment has to be made considering patient's anatomical features and stone peculiarities (such as size and location). Our study concludes that rigid and flexible ureteroscopy (URS/RIRS) is a reliable technique for treatment of $<2 \mathrm{~cm}$ urinary stones in paediatric age group. It shows low rate of major complications and promising results in terms of stone-free rate. We hope that more and more integration will be possible between adult and paediatric urologists to make endourological procedures more familiar to the latter.

\section{References}

1. Bowen DK, Tasian GE. Pediatric Stone Disease. Urol Clin North Am. 2018; 45:539-550.

2. EAU Guidelines. Edn. presented at the EAU Annual Congress Amsterdam 2020. ISBN 978-94-92671-07-3. 
3. Atan A, Balci M. Medical expulsive treatment in pediatric urolithiasis. Turk J Urol. 2015; 41:39-42.

4. Samotyjek J, Jurkiewicz B, Krupa A. Surgical treatment methods of urolithiasis in the pediatric population. Dev Period Med. 2018; 22:88-93.

5. Kilıç Ö, Akand M, Van Cleynenbreugel B. Retrograde intrarenal surgery for renal stones - Part 2. Turk J Urol. 2017; 43:252-260.

6. Whatley A, Jones P, Aboumarzouk O, Somani BK. Safety and efficacy of ureteroscopy and stone fragmentation for pediatric renal stones: a systematic review. Transl Androl Urol. 2019; 8(Suppl 4):S442-S447.

7. Jaidane M, Hidoussi A, Slama A, et al. Factors affecting the outcome of ureteroscopy in the management of ureteral stones in children. Pediatr Surg Int. 2010; 26:501-4.

8. Azili MN, Ozcan F, Tiryaki T. Retrograde intrarenal surgery for the treatment of renal stones in children: factors influencing stone clearance and complications. J Pediatr Surg. 2014; 49:11615.

9 Erkurt B, Caskurlu T, Atis G, et al. Treatment of renal stones with flexible ureteroscopy in preschool age children. Urolithiasis. 2014; 42:241-5.

10. Ozgur Tan $M$, et al. The impact of radiological anatomy in clearance of lower calyceal stones after shock wave lithotripsy in paediatric patients. Eur Urol. 2003; 43:188.

11. Ather MH, Noor MA. Does size and site matter for renal stones up to 30-mm in size in children treated by extracorporeal lithotripsy? Urology. 2003; 61:212-5.
12. Somani BK, Giusti G, Sun Y, et al. Complications associated with ureterorenoscopy (URS) related to treatment of urolithiasis: the Clinical Research Office of Endourological Society URS Global study. World J Urol. 2017; 35:675-681.

13. Kucukdurmaz F, Efe E, Sahinkanat T, et al. Ureteroscopy with holmium:Yag laser lithotripsy for ureteral stones in preschool children: analysis of the factors affecting the complications and success. Urology. 2018; 111:162-167.

14. Somani BK, Griffin S. Ureteroscopy for paediatric calculi: The twin-surgeon model. J Pediatr Urol. 2018; 14:73-74.

15. Berrettini A, Boeri L, Montanari E, et al. Retrograde intrarenal surgery using ureteral access sheaths is a safe and effective treatment for renal stones in children weighing $<20 \mathrm{~kg}$. J Pediatr Urol. 2018; 14:59.e1- 59.e6.

16. Chu L, Sternberg KM, Averch TD. Preoperative stenting decreases operative time and reoperative rates of ureteroscopy. $J$ Endourol. 2011; 25:751-754.

17. Azili MN, Ozturk F, Inozu M, et al. Management of stone disease in infants. Urolithiasis. 2015; 43:513-519.

18. Aksoy Y, Yapanoglu T, Özbey I. The efficacy and safety of extracorporeal shock wave lithotripsy in children. Eurasian J Med. 2009; 41:120-125.

19. Muslumanoglu AY, Tefekli AH, Altunrende F, et al. Efficacy of extracorporeal shock wave lithotripsy for ureteric stones in children. Int Urol Nephrol. 2006; 38:225-9.

\section{Correspondence}

Stefania Ferretti, MD

sferretti@ao.pr.it

Monica Cuschera, MD

monica.cuschera@hotmail.it

Claudia Gatti, MD

tintswal@libero.it

Riccardo Milandri, MD (Corresponding Author)

riccardomilandri85@gmail.com

Tommaso Bocchialini, MD

tommaso.bocchialini@libero.it

Elisa Simonetti, MD

elisasimonetti88@gmail.com

Pietro Granelli, MD

granellipietro@gmail.com

Umberto Vittorio Maestroni, MD

umaestroni@aopr.it

Via Gramsci 14, 43126 Parma (Italy)

Davide Campobasso, MD

d.campobasso@virgilio.it

Antonio Frattini, MD

antonio.frattini@ausl.re.it

Via Donatori di Sangue, 42016 Guastalla (Italy) 